\title{
THE ROLE OF THE COMPUTED TOMOGRAPHY IN STUDYING OF THE PROGRESSION IN THE PLEURAL EMPYEMA
}

\author{
I. Novakov', G. Hadjigeorgiev², J. Peshev³ ${ }^{3}$ G. Ivanov³ \\ ${ }^{1}$ Department of Thoraco-abdominal Surgery, Medical University; Plovdiv, ${ }^{2}$ Department of \\ Roentgenlogy of Radiology, Medical University; Plovdiv, ${ }^{3}$ Department of Clinical Pathology, \\ Medical University; Plovdiv
}

Reviewed by: Assoc. prof. R. Radev, PhD

\begin{abstract}
Pleural empyema is defined as an infection of the pleural space with the formation of purulent pleural fluid. As an empyema progresses, fibrosis of the pleural space develops. The pleural fibrosis represents the most debilitating aspect of empyema progression. As fibrosis increase, more invasive methods become necessary in treatment of empyema. The aim of this experimental study was to represent opportunities of the computed tomography (CT) in studying empyema progression, especially development of pleural fibrosis. Materials and Methods: Group of six "New-Zealand white" rabbits with induced right pleural empyema was used for this study. On $4^{\text {th }}$ and $12^{\text {th }}$ day of empyema progression, computed tomography of the animal's thorax was performed. Quantitative Image Analysis (Densitometry) of the right pleural space was done. Results: The mean density value on $4^{\text {th }}$ day was $21,32(\mathrm{SD}-1,79)$ Hounsfield units $(\mathrm{HU})$. The pathological changes on $12^{\text {th }}$ day shown mean density value of 55,43 (SD-2, 27) HU. The difference between values on 4 th and $12^{\text {th }}$ day were significant $(\mathbf{p}<0,025)$. Discussion and conclusion: Densitometry changes in the pleural space, establishing with computed tomography, correlate with macroscopic and microscopic changes in empyeama progression. CT is a method for determine the pleural fibrosis in empyema progression. Experimental study confirmed the role of $\mathrm{CT}$ in practice for choosing the method of empyemas treatment.
\end{abstract}

Keywords: empyema, pleural fibrosis, computed tomography

\section{INTRODUCTION}

Pleural empyema is defined as an infection of the pleural space with the formation of purulent pleural fluid. Three stages of empyema progression are accepted - exudative, fibrinopurulent and stage of organization. ${ }^{1}$ Without to put exact border between different stages, they represent the evolution of empyema and determine the methods of its treatment. ${ }^{2,3}$ As an empyema progresses, loculations and fibrosis of the pleural space develop. Pleural loculations and pleural fibrosis require more invasive methods of empyemas' treatment. ${ }^{4}$

The process of pleural fibrosis, even today, represents the most debilitating aspect of pleural empyema. If the fibrosis of the pleural space continues to progress, a fibrothorax may ultimately developed. ${ }^{\mathbf{4 , 5}, 6,7}$

The aim of this experimental study was to represent the opportunities of computed tomography (CT) in studying empyema progression, especially development of pleural

Address for correspondence:

I. Novakov, Dept of Thoraco-abdominal Surgery, Medical University, 15 A, "Vasil Aprilov" Str. 4000, Plovdiv, BULGARIA

E-mail: inovakov2003@yahoo.com fibrosis. We hypothesized that CT, with its opportunities for densitometry, may determine the changes in the pleural cavity in empyema evolution, with development of pleural fibrosis as a final result.

\section{MATERIALS AND METHODS:}

Group of six "New-Zealand White" rabbits with body weight of 2,5 to $3,0 \mathrm{~kg}$. was used for this experimental study. An empyema was induced using a method previously described by us. In brief, a catheter was placed in the right pleural space and sterile pleural effusion was caused by injecting of $0,5 \mathrm{ml}$ turpentine through the catheter. Twenty-four hours after that, $2 \mathrm{ml}$ bacterial culture of Klebsiella pneumoniae $\left(900 \times 10^{6}\right.$ bacteria/ml - standart 3 according to Mc Farland) was injected through the catheter. Twenty-four hours after empyema induction, a diagnostic thoracentesis was performed, in which $2 \mathrm{ml}$ of pleural fluid were removed. The fliud was cultured on a blood agar plate to verify the presence of an empyema. In all the animals bacterial growth of Klebsiella pneumoniae was established, which proved pleural empyema. 


\section{CARE FOR ANIMALS}

Care for animals and their euthanasia were performed according to internationally accepted "Guide of the Care and Use of Experimental Animal Care" and Law for Vet Activity in Bulgaria (An Official Gazette, Issue 87; 2005).

\section{COMPUTED TOMOGRAPHY AND INVESTIGATION PROGRESSION OF EMPYEMA}

We performed computed tomography on the thoracic cavity on $4^{\text {th }}$ and $12^{\text {th }}$ days of empyema progression, using device CYTEC-3000; General Electric. Computed tomography (CT) was performed onto previously anaesthetized animals, which were placed in normal according to the table position. We used the thickness of the cuts of $5 \mathrm{~mm}$, with step also of $5 \mathrm{~mm}$. For every animal was chosen an area of the right pleural space for performing quantitative image analysis (densitometry). This area is also known as an investigated region of interest (ROI). In CT, values of densitometry were measured in Hounsfield units (HU).

\section{MACROSCOPIC AND MICROSCOPIC CHANGES IN EMPYEMA PROGRESSION}

On $12^{\text {th }}$ day of empyema progression autopsy of the animals was performed. In animals placed in supine position, thoracic cage was dissected by removing carefully the sternum. In this way we ensured approach to the right and left pleural cavities, which allow their comparatively studying. Macroscopic changes in the right pleural space were documented by digital photography (device Nikon-Colpix 3200).

The tissue specimens of the right lung with visceral pleura from all animals were fixed in paraffin. Histological specimens were performed from paraffin blocks stained with hematoxilin and eosin. Except this standard staining, we performed staining of the specimens by Van-Gieson method.

\section{STATISTICAL ANALYSIS}

The values of densitometry of the changes of the pleural cavity were calculated automatically by computed tomography device. The device shown the data in imagines as: a size of ROI ( in $\mathrm{Mm}^{2}$ ), the mean density of ROI(in $\mathrm{HU}$ ) with standard deviation (SD).Additionally, using a variation analysis, we calculated the mean value of densitometry (with SD) for all results from $4^{\text {th }}$ day and from $12^{\text {th }}$ day of empyema progression. Results from $4^{\text {th }}$ and from $12^{\text {th }}$ days were compared by t-test of Student-Fisher. Significant difference was accepted with levels of $p<0,05$.

\section{RESULTS}

The results of densitometry of the changes in the right pleural cavity are shown in table 1 .

Table 1: Computed tomography densitometry in empyema progression.

\begin{tabular}{||c|c|c|c|c|c|c||}
\hline \multirow{2}{*}{ rabbit } & \multicolumn{3}{|c|}{ 4th day of empyema } & \multicolumn{3}{|c|}{ 12th day of empyema } \\
\cline { 2 - 8 } & $\begin{array}{c}\text { ROI } \\
(\mathrm{mm} 2)\end{array}$ & $\begin{array}{c}\text { density } \\
\text { of ROI } \\
\text { (HU) }\end{array}$ & SD & $\begin{array}{c}\text { ROI } \\
(\mathrm{mm} 2)\end{array}$ & $\begin{array}{c}\text { density } \\
\text { of ROI } \\
\text { (HU) }\end{array}$ & SD \\
\hline 1 & 8 & 22,1 & 2,4 & 8 & 54,8 & 4,1 \\
\hline 2 & 9 & 21,4 & 2,1 & 10 & 51,4 & 3,2 \\
\hline 3 & 7 & 18,7 & 1,7 & 7 & 50,1 & 3,5 \\
\hline 4 & 11 & 23,6 & 2,5 & 10 & 55,2 & 3,8 \\
\hline 5 & 8 & 19,8 & 1,9 & 8 & 53,3 & 3,4 \\
\hline 6 & 7 & 22,3 & 2,4 & 8 & 55,8 & 4,2 \\
\hline- & - & $\begin{array}{c}\text { mean21, } \\
32\end{array}$ & 1,79 & - & mean & 2,43 \\
\hline \hline
\end{tabular}

As shown from tabl.1, all the ROI were larger than $7 \mathrm{Mm}^{2}$. The mean densitometry of the ROI from $4^{\text {th }}$ day was 21,32 $\mathrm{HU}$ and from $12^{\text {th }}$ day was $53,43 \mathrm{HU}$. There was significant difference between densitometry from $4^{\text {th }}$ and $12^{\text {th }}$ day - level of $p<0,025$.

Imagines from 4th day of empyema progression clearly demonstrated effusion in the right pleural space. Pleural drainage is clearly visible into pleural effusion.(fig.1)

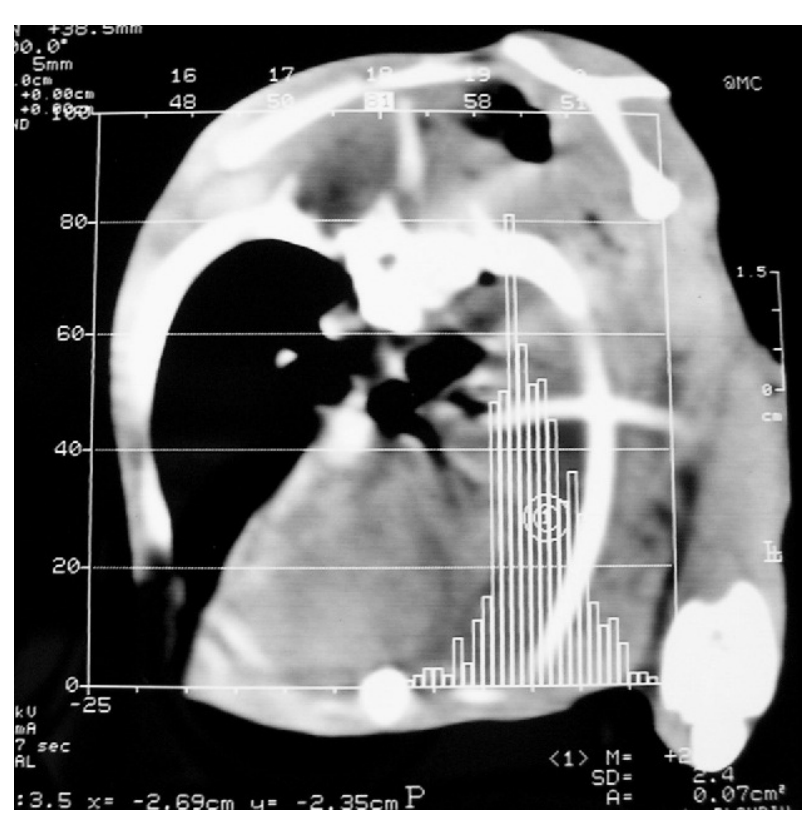

Figure 1: Computed tomography image of $4^{\text {th }}$ day of empyema progression.

(ROI/7 $\mathrm{mm}^{2}$ / with density value of 22,3 HU /SD2,4) 
The imagines from $12^{\text {th }}$ day of empyema progression accept changes in the right pleural space with high value on densitometry (tabl.1), demonstrate the changes in its size. The size of the right pleural space is smaller than left with visible retraction of the intercostal spaces. (fig.2)

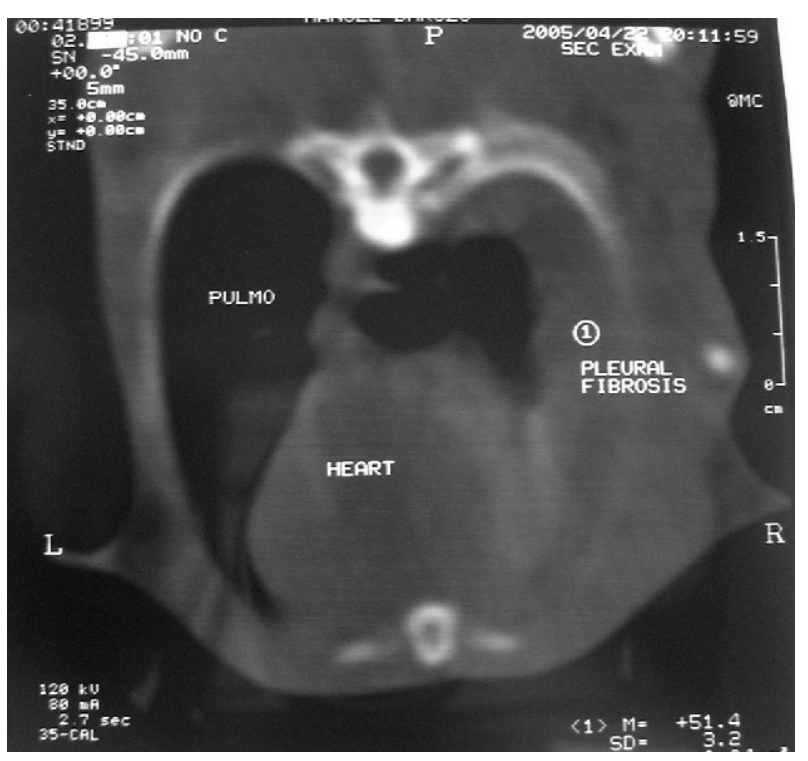

Figure 2: Computed tomography image of $12^{\text {th }}$ day of empyema progression.

(1 points ROI with density value of $51,4 \mathrm{HU} / S D 3,2)$

The changes in the right pleural space, establishing macroscopically, after dissection of the thoracic cage, demonstrate: pleural adhesions, pleural loculations containing fibrinous effusion with high viscosity and retraction of the intercostal spaces (fig.3).

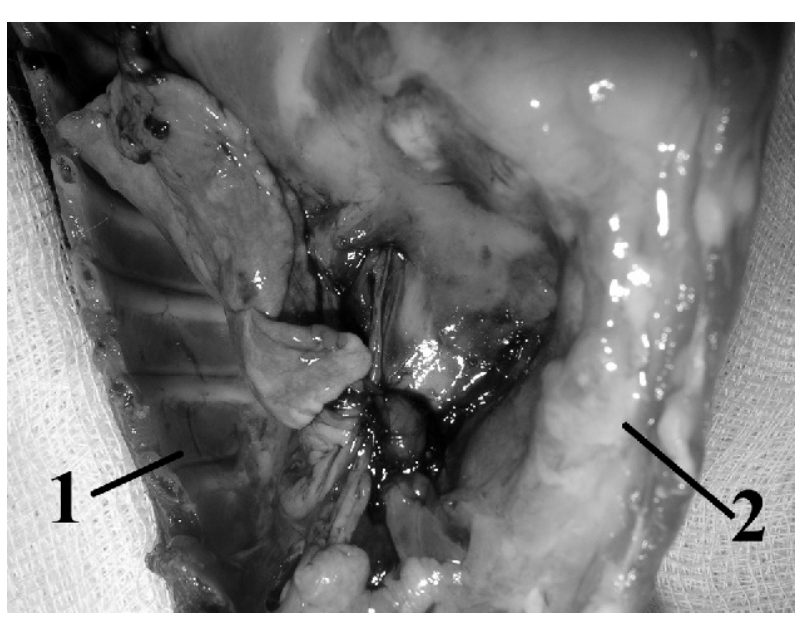

Figure 3: Digital photography of the thorax on $12^{\text {th }}$ day of empyema after removing the sternum. (1 points to the left pleural cavity; 2 points to the right pleural cavity with pleural adhesions and retraction of the intercostal spaces).

Fibrous changed visceral pleura is represented after dissection of the right lung. It blocks expansion of the lung, causing so called trapped lung. (fig.4)

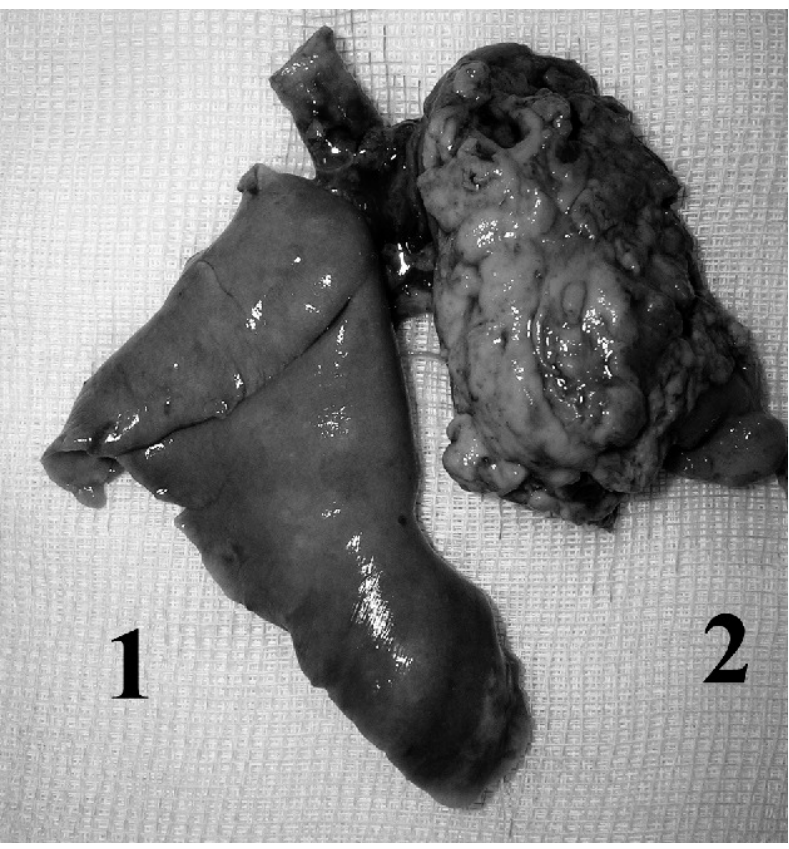

Figure 4: Digital photography of the lungs after dissection of the thorax on $12^{\text {th }}$ day of empyema. (1 points to the normal left lung; 2 points to the trapped

Microscopically, in the visceral pleura from $12^{\text {th }}$ day of empyema progression, is shown proliferation of fibroblasts, synthesis of collagen fibres, pleural infiltration of monocytes and lymphocytes and capillaries as a sign of angiogenesis. The same changes are visible in the lung parenchyma, below mesothelial layer. These changes were established on specimens, staining with hematoxilin and eosin. (fig.5).

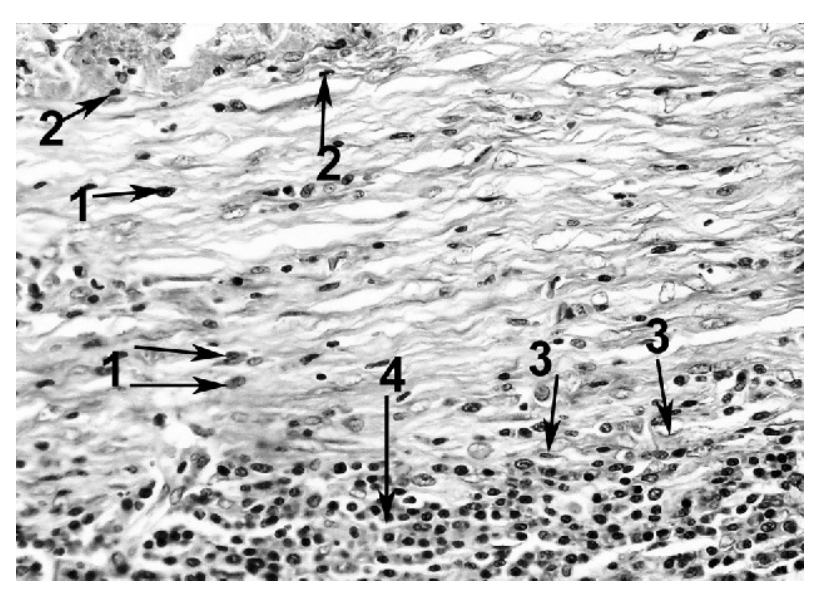

Figure 5 Photomicrograph of thickened visceral pleura and underneathed lung on day 12 of empyema progression $(X 40) . / 1$ points to fibroblasts, 2 points to mesothelial cells, 3 points to endothelial cells and 4 points to mononuclear cells (monocytes, lymphocytes)

Staining the specimens of $12^{\text {th }}$ day of empyema by Van-Gieson, demonstrate abundance of collagen fibres. The collagen fibres are staining in red as it is shown of fig. 6 


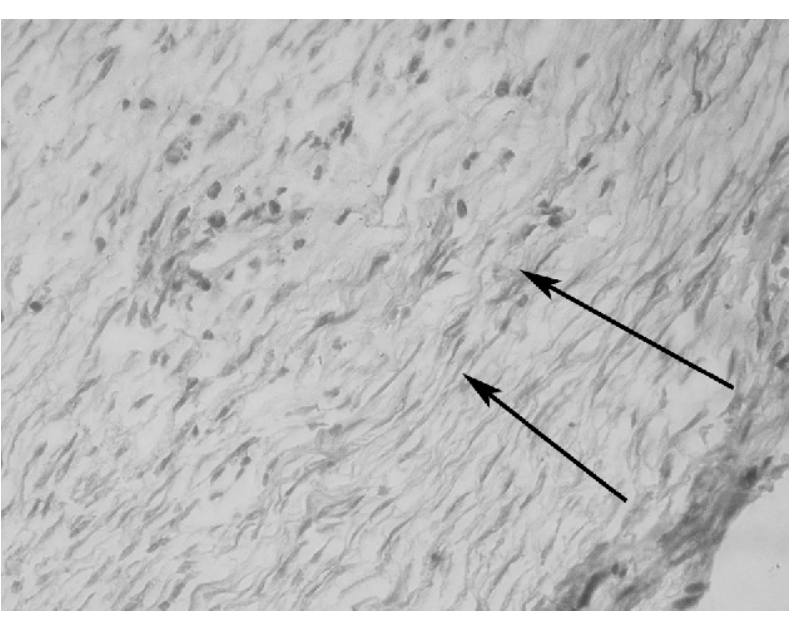

Figure 6 Photomicrograph of Van Gieson,s staining of visceral pleura on day 12 of empyema progression $(X$ 20). Extensive pleural fibrosis is shown. The arrows point to the collagen filaments with red staining.

\section{DISCUSSION}

In this experimental study, we have demonstrated the opportunities of the computed tomography for studying the changes in pleural space which are developed in empyema progression. In our previously study, we had established microscopically the first signs of beginning pleural fibrisis early on $4^{\text {th }}$ day of empyema. ${ }^{8}$ On $12^{\text {th }}$ day were shown macroscopically signs of pleural fibrosis, which had demonsrated stage of organization of empyema. ${ }^{9}$ That is why, on the base of these results, we have choosen $4^{\text {th }}$ and 12 th days of empyema progression for performing the computed tomography imagines. We considered, that investigated region of interest (ROI) must be large enough to increase the accuracy of the mean value of the quantitative imagine analysis (Densitometry). For the small size of the animals, ROI larger than $5 \mathrm{~mm}^{2}$, we consider as enough large. All our measurments were performed onto ROI $>$ of $7 \mathrm{~mm}^{2}$. Differentiation of the pleural effusion as transudate and exudate is done on the base of the results of their biochemical analysis. ${ }^{10,11}$ Protein concentration of the effusion $<30 \mathrm{~g} / \mathrm{l}$ is a sign for transudate and protein $>30 \mathrm{~g} / \mathrm{l}$ is a typical for exudate. It is considered that the computed tomography also can differentiate transudate from exudate using values from densitometry of the effusion. The values of $<$ $18 \pm 2 \mathrm{HU}$ are typical for transudate and for exudate the values of $>18 \pm 2 \mathrm{HU}^{12}$ In our study was establishing mean density value for effusion of $21,32 \mathrm{HU}$ on $4^{\text {th }}$ day of empyema progression. This density value demonstrates that effusion on $4^{\text {th }}$ day is considered as exudate. In empyema progression, the exudate becomes fibrinous, with creating fibrinous peel onto parietal and visceral pleural surfaces. There are pleural adhesions with formation pleural loculations. The process of organization of the pleural fibrinous peel becomes with development of pleural fibrosis. The pleural fibrosis restricts the lung expansion. ${ }^{4,6}$ Our macroscopic results clearly demonstrate the pleural fibrosis on the $12^{\text {th }}$ day of empyema, with formation of the trapped lung. Microscopically presentation of the abundance of collagen fibers confirms the pleural fibrosis. Macroscopically and microscopically changes in the right pleural cavity from $12^{\text {th }}$ day of empyema correlate with the results of computed tomography densitometry. It is acceptable, that density values in range of 50-65 HU are typical for tissue fibrosis. Our computed tomography images from $12^{\text {th }}$ day of empiema have shown mean density value of 53,43 HU. That is why, these results demonstrate the process of pleural fibrosis, establishing after dissection of the thoracic cage. Wegener et al. have shown, that CT have demonstrated the drainage in the pleural cavity, which is frequently difficult on conventional chest radiographs. ${ }^{12} \mathrm{CT}$ has exactly determined the position of the catheter in the pleural space. ${ }^{13}$ The results in this experimental study confirm these opportunities of the CT. All of our images clearly demonstrated the position of the thoracic drainage.

\section{CONCLUSION}

1. This experimental study has shown, that CT with opportunities for densitometry of the pleural effusions may exactly differentiate these with characteristic of exudate.

2. CT, with its opportunities to determine the changes in pleural space in empyema progression, is a method, which may used for experimental study the pleural fibrosis.

3. Experimental results confirm that $\mathrm{CT}$ is a method using in practice for empyema diagnostic and choosing the method of its treatment.

\section{REFERENCES}

1. Andrews NC, Parker EF, Shaw RR et al. Management of nontuberculous empyema. Am Rev Respir Dis, 1962;85:935-936

2. Light RW. Parapneumonic effusions and empyema. Pleural diseases. $4^{\text {th }}$ ed. Philadelphia PA: Lippincott, Williams and Wilkins, 2001; 151-181.

3. Light RW.Parapneumonic effusions and empyema.Proc AmThorac Soc, 2006;3:75-80

4. Sasse SA, Jadus MR, Kukes GD. Pleural fluid transforming growth factor-b1 correlates with pleural fibrosis in experimental empyema. Am J Respir Crit Care Med; 2003; 168: 700-705.

5. Hage CA, Mihammed KA, Antony VB. Phatogenesis of pleural infection. Respirology, 2004;9:12-15

6. Doelkin P.,Sahn SA. Trapped Lung. Sem. in Resp. and Crit Care Med,2001;6:631-635.

7. Mutsaers SE et al. Pathogenesis of pleural fibrosis. Respirology,2004;9:428-440.

8. Novakov IP et al. Experimental pleural empyema in rabbits-cellular and biochemical changes. Trakia J. Scien., 2005;1:26-29

9. Novakov IP et al. Experimental pleural empyema - pathologic changes. Trakia J Scien, 2005;2:61-65 
The role of the computed tomography in studying of the progression in the pleural empyema

10. Light RW. Diagnostic approach in a patient with pleural effusion. Eur Respir Mon, 2002; 22:131-145.

11. Light RW, MacGregor MI, Luchsinger PC et al. Pleural effusions: the diagnostic separation of transudates and exudates. Ann Inter Med 1972; 77:507-513
12. Wegener $\mathrm{OH}$. Whole Body Computed Tomography. $2^{\text {th }}$ ed. Blackwell Scientific Publications, 1992; 225-229.

13. Kerr A, Vasudevan VP, Powell S et al. Percutaneous catheter drainage for acute empyema.NY State J Med,1991;91:4-7 